Research Paper

\title{
Cryptotanshinone Suppresses Non-Small Cell Lung Cancer via microRNA-146a-5p/EGFR Axis
}

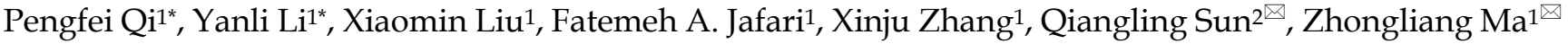 \\ 1. Lab for Noncoding RNA \& Cancer, School of Life Sciences, Shanghai University, Shanghai, China \\ 2. Department of Thoracic Surgery, Shanghai Chest Hospital Affiliated to Shanghai Jiao Tong University, Shanghai, China \\ ${ }^{*}$ Co-first authors with equal contribution to the manuscript \\ $\square$ Corresponding authors: zlma@shu.edu.cn, shqling@163.com; Address: Room 107, School of Life Sciences, No.381, Nanchen Road, Baoshan District, Shanghai, \\ China \\ (c) Ivyspring International Publisher. This is an open access article distributed under the terms of the Creative Commons Attribution (CC BY-NC) license \\ (https://creativecommons.org/licenses/by-nc/4.0/). See http://ivyspring.com/terms for full terms and conditions.
}

Received: 2018.11.06; Accepted: 2019.01.28; Published: 2019.04.22

\begin{abstract}
Epidermal growth factor receptor (EGFR), a cancer-driven gene, plays an important role in tumorigenesis of lung cancer. Cryptotanshinone (CT) is the main constituent of salia miltiorrhiza and has been found to affect tumor progression. However, the mechanism of CT on lung cancer is still not clear. Here we found that CT could suppress the proliferation of non-small cell lung cancer (NSCLC) by inhibiting EGFR. We further confirmed that knockdown of EGFR also suppressed cell proliferation and arrested cell cycle progression. Furthermore, we evaluated EGFR was a direct target gene of miR-146a-5p which was upregulated by CT. In general, our results proved that CT could restrain NSCLC via miR-146a-5p/EGFR axis. CT and miR-146a-5p have the potential to be positive candidates in drug development of NSCLC.
\end{abstract}

Key words: NSCLC, cryptotanshinone, EGFR, miR-146a-5p

\section{Introduction}

Lung cancer is the most deadly malignancy in the last decade, accounting for about 1.6 million deaths every year globally [1-3]. Almost $85 \%$ of lung cancers are non-small cell lung cancers (NSCLC), and 5 -year survival rate is about $15 \%[4,5]$.

Epidermal growth factor receptor (EGFR), a receptor tyrosine kinase, plays important roles in cell proliferation, survival, migration and apoptosis, and has been found to be positively involved in tumorigenesis $[6,7]$. Overexpression or mutation of EGFR can be observed in about $30 \%$ of solid tumors [8], including glioblastoma [9], lung cancer [10] and breast cancer [11]. EGFR-targeted therapy is the most promising treatment for NSCLC currently [12-14], and up to now three generations of EGFR tyrosine kinase inhibitors (EGFR-TKIs) have been developed and applied [15-18]. However, with the progression of treatment, the emergence of drug resistance has become a tough problem [19-22]. EGFR L858R/ T790M/C797S mutations can develop resistance to almost all existing EGFR-TKIs [23]. Therefore, it is urgent to find a new EGFR target for lung cancer treatment.

Tanshinones are the main functional components of salvia miltiorrhiza which is widely used in traditional Chinese herbal medicine for many years [24]. Cryptotanshinone (CT), tanshinone IIA (T2A) and tanshinone I (T1) are liposoluble monomers of Tanshinones [21]. It has been found that CT could restrain prostate carcinoma, rhabdomyosarcoma and breast carcinoma cells by inhibiting the phosphorylation of Retinoblastoma and downregulating the expression of cyclin D1, which may be related to suppression of mammalian target of mTOR signaling pathway by CT [25]. Furthermore, some studies have found that CT could inhibit lung cancer by down-regulating the IGF-1R/PI3K/Akt signaling pathway and promoting pro-death autophagy [26, 27], and could reverse cisplatin resistance in lung cancer cells [28]. Our hypothesis is that CT can inhibit lung cancer via suppressing EGFR.

In our previous work, we found that tanshinones can upregulate some microRNAs (miRNAs), such as miR-32 and miR-137 [29, 30]. MiRNAs, a kind of 
noncoding RNA, can specifically bind with their target genes to regulate their expression and cause tumorigenesis [31-33]. In addition, it has been found that some miRNAs could inhibit EGFR signaling pathway and provide candidates for EGFR-targeted therapy [32, 34, 35]. Moreover, studies have shown that miR-146a-5p can influence prostate cancer therapy and inhibit apoptosis of breast cancer cells by FOXP3-miR-146a-NF-kB axis [36]. Zhang et al. showed miR-146a-5p plays a vital role in lung carcinogenesis [37]. Here we verified that CT can inhibit NSCLC via miR-146a-5p/EGFR axis. CT and miR-146a-5p have the potential to be positive candidates in drug development of NSCLC.

\section{Material and Methods}

\section{Chemicals and reagents}

CT was obtained from Haoxuan Biotech (Xi'an, China) and dissolved with dimethyl sulfoxide (DMSO). Solid biochemical reagents were mainly purchased from Sangon Biotech (Shanghai, China). Liquid biochemical reagents were mainly obtained from Sinopharm Chemical Reagent (Shanghai, China).

\section{Cell culture}

The HCC827, H1299, H1975, A549 and HEK293T NSCLC cell lines were obtained from the American Type Culture Collection (ATCC, USA) and the cell bank of China Academy of Sciences (Shanghai, China). The H1299, H1975, HCC827, A549 and HEK293T cells were cultured in DMEM or RPMI-1640 medium (Corning, USA) with $1 \%$ penicillinstreptomycin solution (HyClone, USA) and 10\% fetal bovine serum which is made in America (FBS, Gibco, USA). Cells were cultured in 5\% carbon dioxide cell incubator at $37^{\circ} \mathrm{C}$.

\section{Cell transfection}

The chemically synthesized negative control miR-mimic (NC), miR-146a-5p mimic, negative control siRNA (siNC) and siEGFR were obtained from Ribobio (Guangzhou, China). At 80\% of H1299 and HCC827 cells confluence, lipofectamine 2000 (Thermo, USA) was used to help transiently synthetics transfection. After transfection 24 to $48 \mathrm{~h}$, subsequent experiments were performed in treated cells, such as cell cycle analysis and western blot.

\section{Cell proliferation, colony formation and cell cycle analysis}

Cells were counted with blood counting chamber after transiently transfected, then 2000 and 600 cells per well were used for cell counting kit- 8 (cck-8) and colony formation assays respectively. Cell cycle analysis, $1 \times 10^{5}$ cells were collected and absolute ethyl alcohol was added slowly to reach $70 \%$ and were stored at $-20^{\circ} \mathrm{C}$ overnight. The cells were precipitated with $25 \mu$ of FBS, suspended with RNase A $(100 \mathrm{ng} / \mathrm{mL})$ for $25 \mathrm{~min}$ at $37^{\circ} \mathrm{C}$, and dyed with PI (50 ng/mL) at $4{ }^{\circ} \mathrm{C}$ for $15 \mathrm{~min}$. A MoFlo XDP flow cytometer (Beckman Coulter, USA) was used to analyze cell cycle distribution of samples and Flow Jo software was used to analyze data. Each experiment was repeated at least three times independently.

\section{RNA extraction and quantitative RT- PCR}

TRIzol reagent (Transgen, Shanghai, China) was used to extract total RNA from treated cells, then PrimeScriptTM RT reagent Kit (Takara, Japan) was used for mRNA reverse transcription and QuantiMir cDNA Kit (Transgen) was used for miRNA reverse transcription. RealStar Green Fast Mixture (GenStar, China) was used to carry out quantitative RT-PCR and data results were analyzed by t-test method. The endogenous controls we selected for mRNA and miRNA were $18 \mathrm{~S}$ rRNA and U6 snRNA respectively and primer sequences used are shown in Table S1.

\section{Nuclear protein extraction, protein extraction and immunoblotting}

Cells were collected and suspended with lysis buffer containing protease inhibitor, blown up and down for $10 \mathrm{~min}$, then centrifuged at maximum speed for $10 \mathrm{~min}$ at $4{ }^{\circ} \mathrm{C}$. Save supernatant (cytosolic fraction) if desired, washed sediment with $500 \mu \mathrm{L}$ ice-cold lysis buffer. Then added 3 folds volumes nuclear extraction buffer for $30 \mathrm{~min}$ on ice, centrifuged at maximum speed for $10 \mathrm{~min}$ at $4{ }^{\circ} \mathrm{C}$ and saved supernatant at $-80{ }^{\circ} \mathrm{C}$. Total protein was extracted and quantified, then we used SDS-page to resolve protein $(30 \mu \mathrm{g})$ and transferred it to PVDF membranes (GE Healthcare, USA). The membrane was blocked with $5 \%$ powdered milk in room temperature for $1 \mathrm{~h}$, then incubated with primary antibody (1:1000) at $4^{\circ} \mathrm{C}$ overnight. After washed with TBST three times and incubated with a secondary antibody (1:10000, CST, USA) for $2 \mathrm{~h}$, we used a chemiluminescence developer (Millipore, USA) to detect protein bands and imaged by chemiluminescence image analysis system (Tanon, Shanghai, China). Each experiment was repeated at least three times independently.

\section{Dual luciferase reporter assay}

According to our prediction, the 3'-UTR of EGFR which contains the predicted binding site of miR-146a-5p, and 3'-mUTR of EGFR, the predicted binding site was point mutated, were respectively inserted into the pGL3 vector (Promega, USA) with molecular cloning techniques, and named pGL3- 
EGFR WT 3'-UTR (EGFR-3'-UTR) and pGL3-EGFR mut 3'-UTR (EGFR-3'-mUTR). Then NC or miR-146a$5 p$ mimic, renilla luciferase plasmid ( $p R L$, Promega), and 24 well plate number of 50\% confluent HEK293T cells were transiently co-transfected with EGFR-3'UTR or EGFR-3'-mUTR vector. $48 \mathrm{~h}$ after transfection, an Orion II Micro-plate Illuminometer (TitertekBerthold, USA) was used to detect luciferase activity of cells. The difference of relative activity was identified by t-test.

\section{EGFR rescue assay}

NSCLC cells were transiently co-transfected with p2k7 of EGFR expression vector (p2k7-EGFR) or p2k7-GFP as well as miR-146a-5p mimic or NC mimic using lipofectamine 2000. $24 \mathrm{~h}$ after transfection, transfection effects were detected by qRT-PCR and western blot. Subsequently, related functional experiments were carried out. Each experiment was repeated at least three times.

\section{Statistical analysis}

The experimental data was expressed as group mean value \pm SEM, differences were evaluated by using t-tests for 2-group comparison, and figures were made using GraphPad Prism 5 software. Differences were considered statistically significant when $p<0.05$.

\section{Results}

\section{CT suppresses expression of EGFR in NSCLC cells}

In our previous work, we have validated that tanshinones could target AURKA in NSCLC cell lines
[29]. We first verified the effect of CT on NSCLC cells, and we treated cells with $5 \mu \mathrm{M}$ CT and detected cell proliferation after $24 \mathrm{~h}, 48 \mathrm{~h}$, and $72 \mathrm{~h}$, and cell proliferation was significantly inhibited in H1299, HCC827 and A549 cells (Figure 1A-C). And then we explored the mechanism that tanshinones inhibited NSCLC, we found that EGFR could be a key cancer-driven gene which is associated with CT. We measured the expression of EGFR in cells after incubated with $5 \mu \mathrm{M}$ CT $48 \mathrm{~h}$. The data showed that the mRNA level of EGFR in H1299 and HCC827 cells was significantly decreased compared with control group (DMSO for $48 \mathrm{~h}$ ) (Figure 1D) and the protein expression level was also downregulated (Figure 1E-F). The results suggested that $\mathrm{CT}$ may affect NSCLC by inhibiting the expression of EGFR.

\section{Downregulation of EGFR suppresses cell proliferation and cell cycle}

In order to study the function of EGFR in NSCLC, EGFR was knocked down by siRNA transfection in H1299 cells. After transfection with siEGFR, the mRNA level of EGFR had almost 50\% decrease compared to the control (Figure 2A). After transfection, the protein level of EGFR was also significantly decreased (Figure 2B-C). Then associated phenotypic experiments were performed in H1299 cells, and results indicated that downregulation of EGFR inhibited cell proliferation and clone formation, as well as arrested the cell cycle progression (Figure 2D-F). These results demonstrated that downregulation of EGFR had the similar effects of CT treatment in NSCLC cells.
A
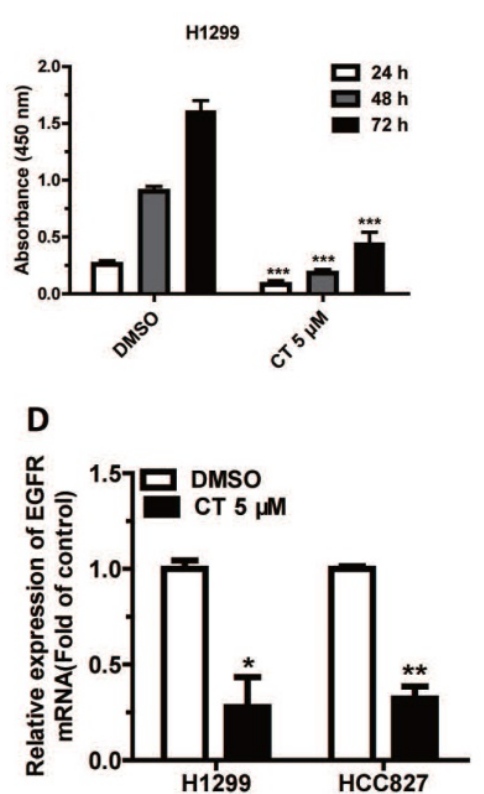

B

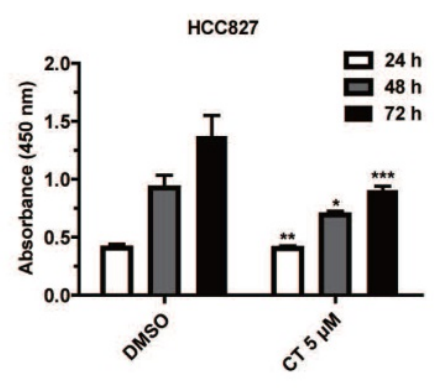

E

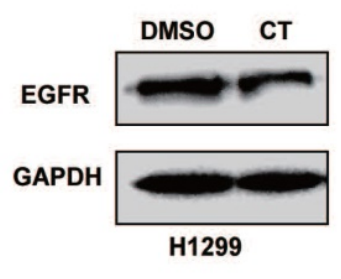

C

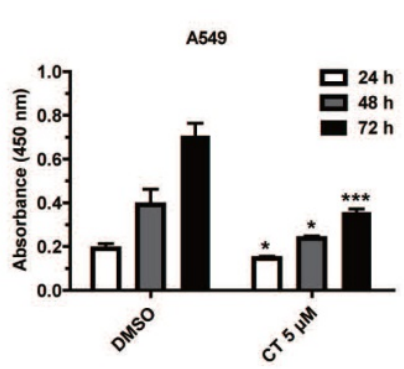

$\mathbf{F}$

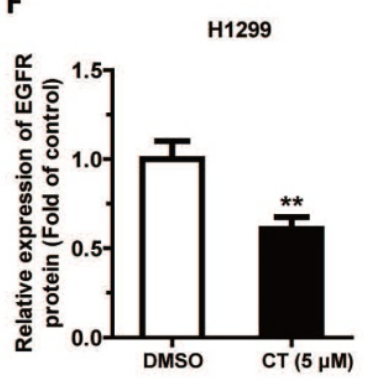

Figure 1. CT could suppress the expression of EGFR: (A-C) Cell Counting Kit-8 assay was used to measure the proliferation of $\mathrm{H} 1299$, HCC827 and A549 cells following treated with $5 \mu M$ CT. (D-F) The expression of the EGFR in $\mathrm{H} 1299$ and $\mathrm{HCC} 827$ Cell lines treated with $5 \mu$ M CT were verified by qRT-PCR $(n=4)$ analysis and by western blot analysis. Data are presented as mean \pm sd and were analyzed by Student's t-test $(n=4, * P$ $<0.05, * * \mathrm{P}<0.01)$. 
A

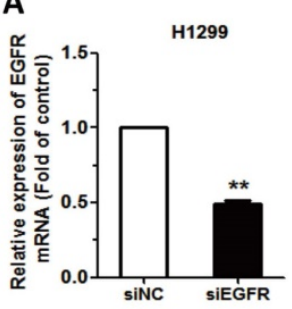

$\mathbf{E}$

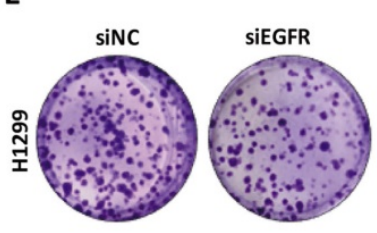

B

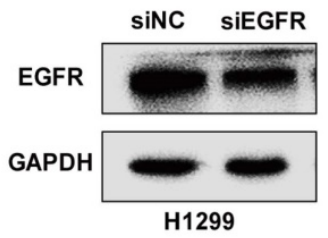

H1299

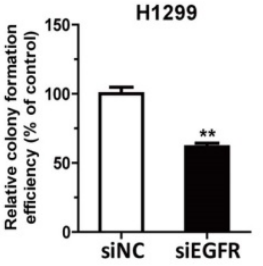

C

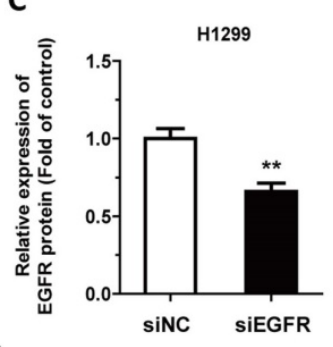

$\mathbf{F}$

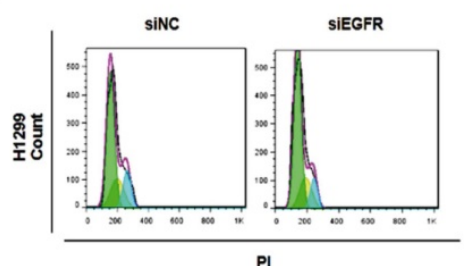

D
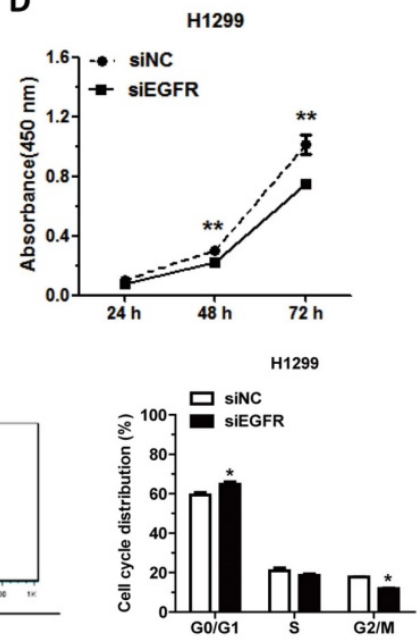

Figure 2. Downregulation of EGFR suppresses cell proliferation and cell cycle progression: (A-C) The mRNA levels of EGFR were detected by qRT-PCR and protein levels were detected by western blot in H1299 cells transfected with siEGFR. (D) CCK-8 assay was used to measure the proliferation of H1299 cells following transfection with siRNA. (E) Colony formation assay in $\mathrm{H} 1299$ cells transfected with siRNA. (F) The cell-cycle distributions of $\mathrm{H} 1299$ cells transfected with siRNA were detected by flow cytometry. Data are presented as mean $\pm \mathrm{sd}$ and were analyzed by Student's t-test $(\mathrm{n}=4, * \mathrm{P}<0.05$, $* * \mathrm{P}<0.01)$.

\section{EGFR is a direct target of miRNA-146a-5p}

In this study, we tried to figure out whether some miRNAs are involved in CT's regulation through targeting EGFR. According to Targetscan (http://www.targetscan.org/vert_72/) software prediction of EGFR, we found miR-146a-5p might potentially target EGFR. Subsequently we used miRTarBase (http://mirtarbase.mbc.nctu.edu.tw/ php/index.php), TargetScan, miRPathDB (https:// mpd.bioinf.uni-sb.de/overview.html), and miRWalk 3.0 (http://mirwalk.umm.uni-heidelberg.de/) to predict target genes of miR-146a-5p, and EGFR was selected (Figure 3A). Then, EGFR 3'-UTR vector and EGFR 3'-mUTR were transfected into H1299 cells as descried previously to verify whether EGFR is a target gene of miR-146a-5p (Figure 3B). The relative luciferase activity in HEK293T cells which were co-transfected with miR-146a-5p mimic, EGFR 3'-UTR and $\mathrm{pRL}$ vector, had a significant decrease compared to control (Figure 3C). While, after co-transfection with miR-146a-5p, EGFR 3'-mUTR and pRL vector, the relative luciferase activity in HEK293T cells did not have significant change (Figure 3C). We also detected that the expression level of EGFR mRNA and protein were significantly downregulated after transfection with miR-146a-5p mimic in H1299 and HCC827 cells (Figure 3D-F). All results showed that EGFR was a direct target of miR-146a-5p.

\section{CT upregulates miRNAs which function as tumor-suppressing genes}

The expression of EGFR-related miRNAs, miR-107-5p, miR-146a-5p and miR-34a, were measured after CT incubation. Data showed the expression of miR-107-5p, miR-146a-5p and miR-34a were significantly increased in H1299 and HCC827 cells (Figure 4A-B). The function of miR-107-5p and miR-34a has been confirmed in our previous studies $[32,38]$. We then plotted the survival curve of lung cancer patients with different miR-146a-5p expression through Kaplan-Meier Plotter website (http:// kmplot.com/analysis/index.php?p=service), the result showed that patients with higher miR-146a-5p expression had better prognosis and suggested that miR-146a-5p might be a potential tumor suppressor (Figure 4C). Moreover, we suspected CT suppressed EGFR expression partly through increasing the expression of miR-146a-5p.

\section{MiR-146a-5p inhibits cell proliferation and arrests cell cycle}

To explore the influence of miR-146a-5p on NSCLC, miR-146a-5p mimic/NC were transfected into H1299 and HCC827 cells, and lipofectamine 2000 was used as a transfection aid. qRT-PCR analysis showed that transfection of miR-146a-5p mimic indeed increased the level of miR-146a-5p (Figure 5A). CCK-8 and colony formation assays showed that cell proliferation was significantly inhibited after transfection with miR-146a-5p mimic in both cell lines (Figure 5B-E). The significant restraining of cell proliferation suggested that $\operatorname{miR}-146 a-5 p$ was a negative regulator of NSCLC. Furthermore transfected with miR-146a-5p mimic induced G0/G1 cell-cycle arrest in both cell lines, which might be one of the mechanisms of cell proliferation inhibition (Figure 5F). Collectively, the results indicated miR146a-5p could significantly inhibit the development of lung cancer. 


\section{Ectopic expression of EGFR promotes cell proliferation and cell-cycle progression}

To verify the negative correlation between miR-146a-5p and EGFR, we ectopically expressed EGFR to test whether it can reverse the inhibitory effect of miR-146a-5p mimic in NSCLC cells. We set up four experimental groups as follows, H1299 cells were co-transfected with p2k7-EGFR or p2k7-GFP and miR-146a-5p mimic or NC, individually named "NC+p2k7-GFP", "miR-146a-5p mimic+p2k7-GFP", "NC+p2k7-EGFR" and "miR-146a-5p mimic+p2k7EGFR". The results showed that the expression of miR-146a-5p was upregulated significantly in H1299 cells transfected with miR-146a-5p mimic (Figure 6A). And the transfection of p2k7-EGFR did not significantly affect the level of miR-146a-5p (Figure
6A). Consistent with previous results, the mRNA and protein level of EGFR were significantly decreased in cells co-transfected with miR-146a-5p mimic and p2k7-GFP (Figure 6B-D). Meanwhile, the mRNA and protein level of EGFR were significantly increased in H1299 cells co-transfection with NC or miR-146a-5p mimic and p2k7-EGFR, compared with cotransfection with NC and p2k7-GFP (Figure 6B-D). Proliferation and cell cycle were also detected after overexpressing EGFR ectopically. The data indicated that overexpression of EGFR completely reversed the suppressive function of miR-146a-5p mimic (Figure $6 \mathrm{E}-\mathrm{G})$. In conclusion, our results suggested that EGFR could promote NSCLC by activating cell proliferation and cell cycle progression.
A
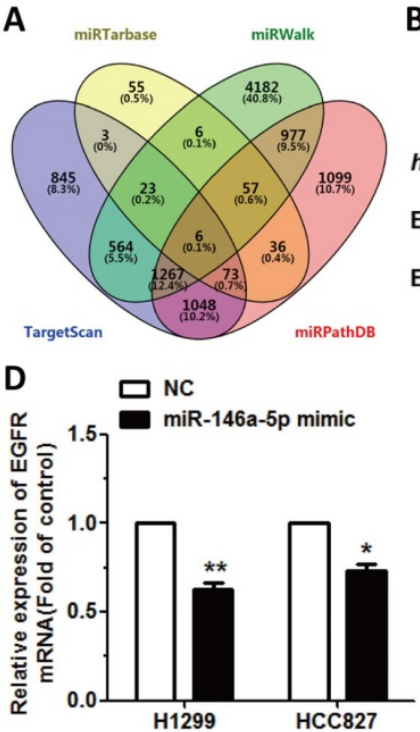

B

hsa-miR-146a-5p $3^{\prime}$ UUGGGUACCUUAAGUCAAGAGU 5 EGFR 3'UTR WT 5' UGUGCUUCCCAAAAUAGUUCUCA 3' EGFR 3'UTR MUT 5 ' UGUGCUUCCCAAAAUUGAUGUGA 3'

E

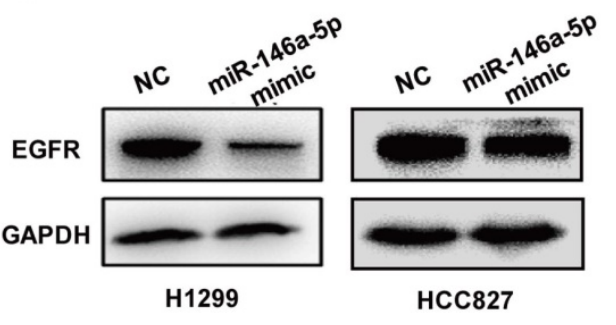

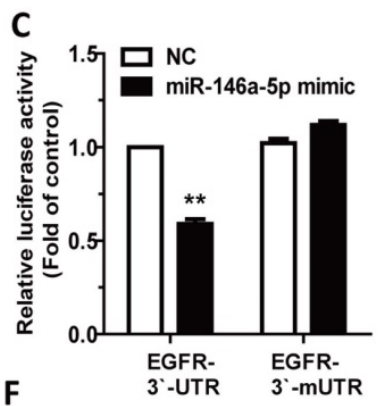

$\mathbf{F}$

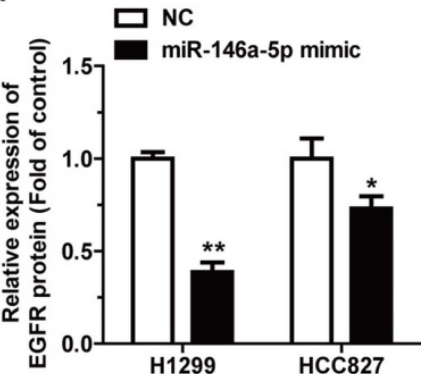

Figure 3. EGFR is a direct target of miR-146a-5p: (A) miRWalk 2.0, miRPathDB, TargetScan and miRTarBase were used to predict potential targets for miR-146-5p. (B) EGFR WT 3'-UTR contains predicted miR-146a-5p binding site. The data show alignment of miR-146a-5p with EGFR WT 3'-UTR and arrows indicate mutagenesis nucleotides. (C) Dual luciferase reporter assay. EGFR-3'-UTR and EGFR-3'-mUTR were co-transfected with miR-146a-5p mimic in HEK293T cells. Data displayed are relative firefly luciferase expression, normalized to Renilla luciferase expression. (D-F) The mRNA levels of EGFR were detected by qRT-PCR and protein levels were detected by western blot in H1299 and HCC 827 cells transfected with miR-146a-5p mimic. Data are presented as mean $\pm s d$ and were analyzed by Student's t-test $(n=3, * P<0.05, * * P<0.01)$.

A

H1299

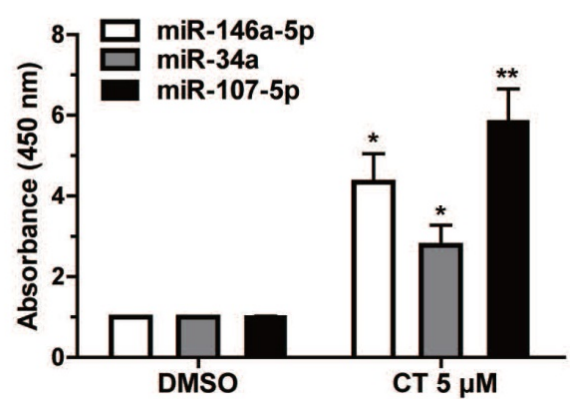

B

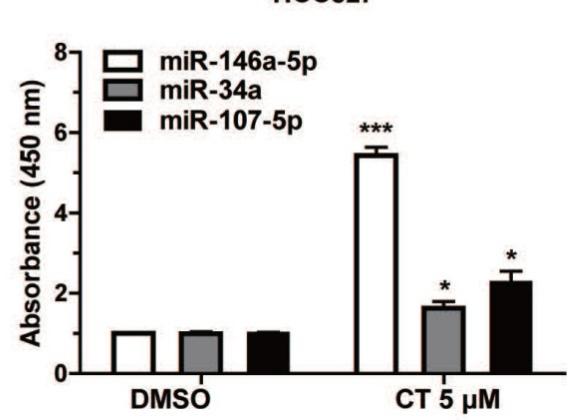

C

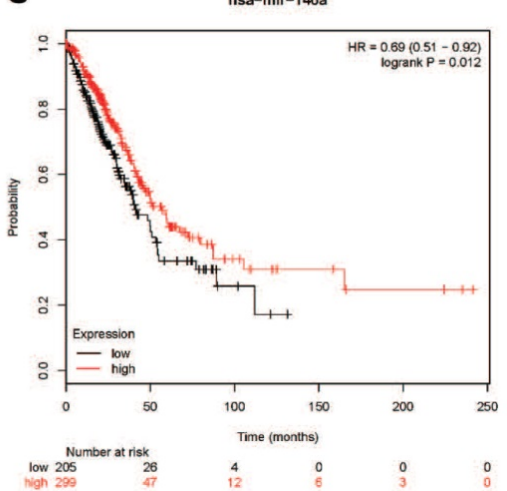

Figure 4. CT could upregulate some miRNAs in NSCLC cells: (A-B) Expression of miR-146a-5p, miR-34a and miR-107-5p in H1299 and HCC827 Cell lines treated with $5 \mu$ M CT were verified by qRT-PCR analysis. Data are presented as mean \pm sd and were analyzed by Student's t-test $(n=4, * P<0.05$, **P $<0.01$, *** $P<0.001)$. (C) The survival curve of lung cancer patients with different miR-146a-5p expression through Kaplan-Meier Plotter website. 
A

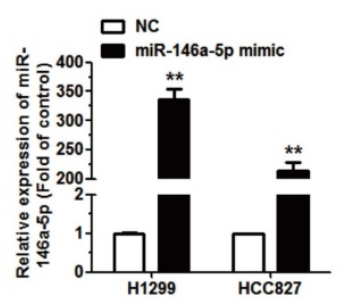

B

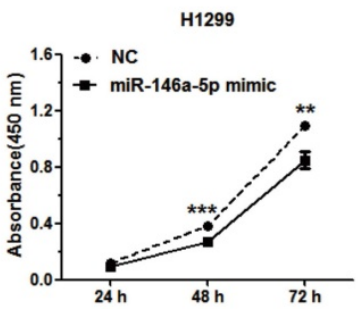

C

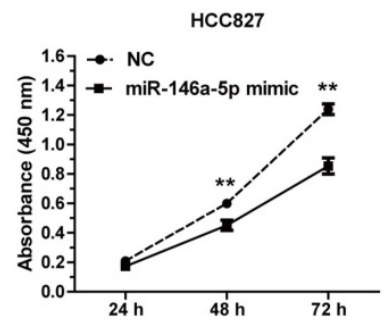

D

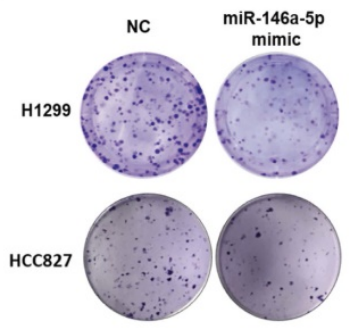

E

HCC827
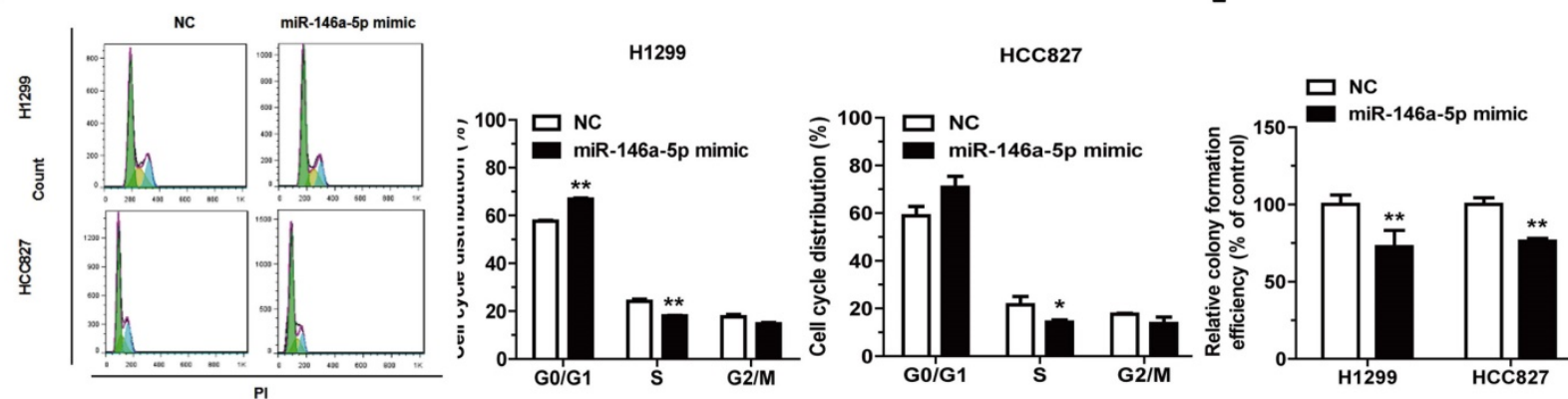

Figure 5. MiR-146a-5p could inhibit cell proliferation and impede cell-cycle progression in NSCLC cells. (A) Upregulation of miR-146a-5p following transfection with $100 \mathrm{nM}$ miR-146a-5p mimic in H1299 and HCC827 cells $(n=4)$. (B-C) Cell Counting Kit-8 (CCK-8) assay was used to measure the proliferation of H1299 and HCC827 cells following transfection with miR-146a-5p mimic. (D-E) Colony formation assay in $\mathrm{H} 1299$ and HCC827 cells transfected with miR-146a-5p mimic. (F) The cell cycle distributions of H1299 and HCC 827 cells transfected with miR-146a-5p mimic were detected by flow cytometry. Data are presented as mean $\pm s d$ and were analyzed by Student's $t$-test $(n=4, * P<0.05$, $* * \mathrm{P}<0.01)$.

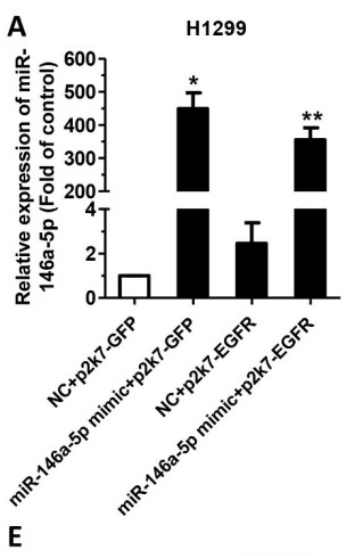

E

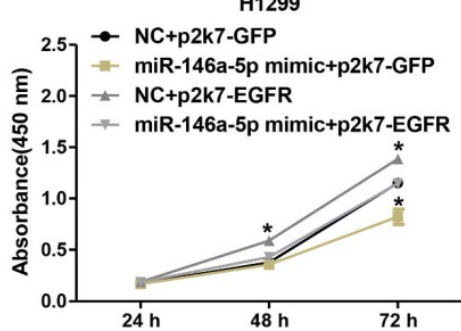

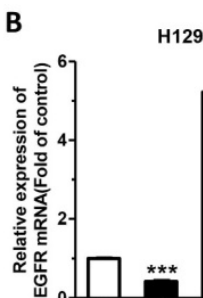
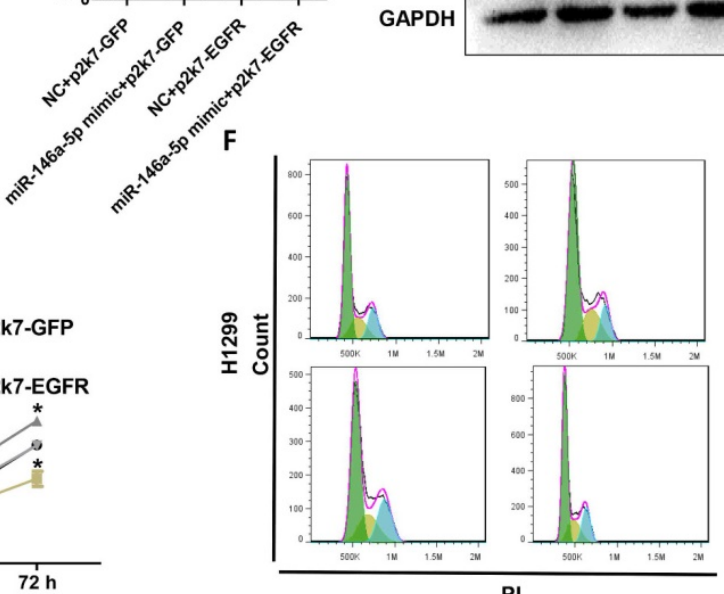

C

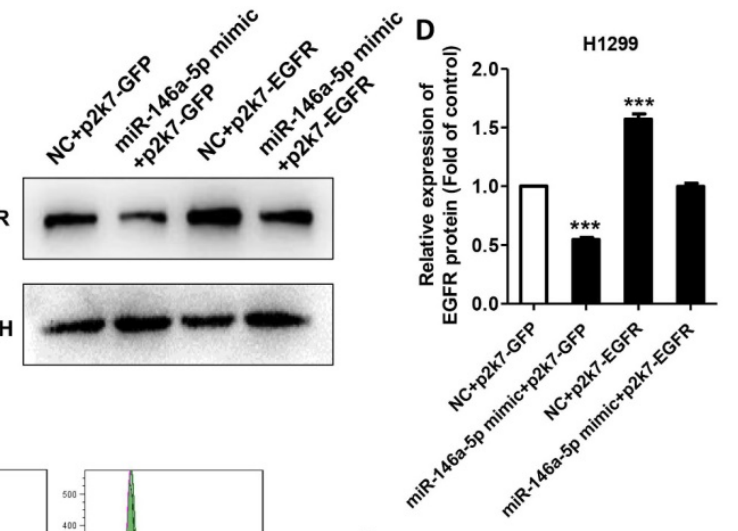

$\mathbf{G}$

H1299

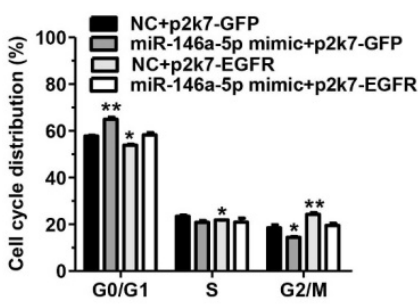

Figure 6. EGFR could rescue the effects of miR-146a-5p in NSCLC cell lines: (A) The levels of miR-146a-5p in H1299 cells following co-transfection with p2k7-EGFR/p2k7-GFP and miR-146a-5p mimic/NC. (B-D) The levels of EGFR mRNA and protein in H1299 cells following co-transfection with p2k7-EGFR/p2k7-GFP and miR-146a-5p mimic/NC. (E) The proliferation of $\mathrm{H} 1299$ cells following co-transfection with p2k7-EGFR/p2k7 and miR-146a-5p mimic/NC, as measured by CCK-8 assay. (F-G) The cell-cycle distributions of $\mathrm{H} 1299$ cells were co-transfected with p2k7-EGFR/p2k7-GFP and miR-146a-5p mimic/NC were detected by flow cytometry. Data are presented as mean \pm sd and were analyzed by Student's t-test $(\mathrm{n}=3, * \mathrm{P}<0.05$, $* * \mathrm{P}<0.01)$.

\section{Discussion}

In recent years, more and more tanshinone drugs are developed for the treatment of anti-bacterial, anti-inflammatory and angina pectoris [39]. In our previous study, we tried to explore the antitumor function of tanshinone monomers in vivo. We treated xenograft tumors in mice by intragastric administration of $\mathrm{T} 1$ and $\mathrm{T} 2 \mathrm{~A}$, and the data proved that $\mathrm{T} 1$ 
and T2A significantly inhibited tumor growth. Herein, we found that CT which significantly suppressed NSCLC and CT might be an efficient candidate for the drug development of NSCLC. Then, we further proved the interaction among CT, miRNAs and EGFR, and defined the relationship between EGFR and miR-146a-5p in NSCLC (Figure 7).

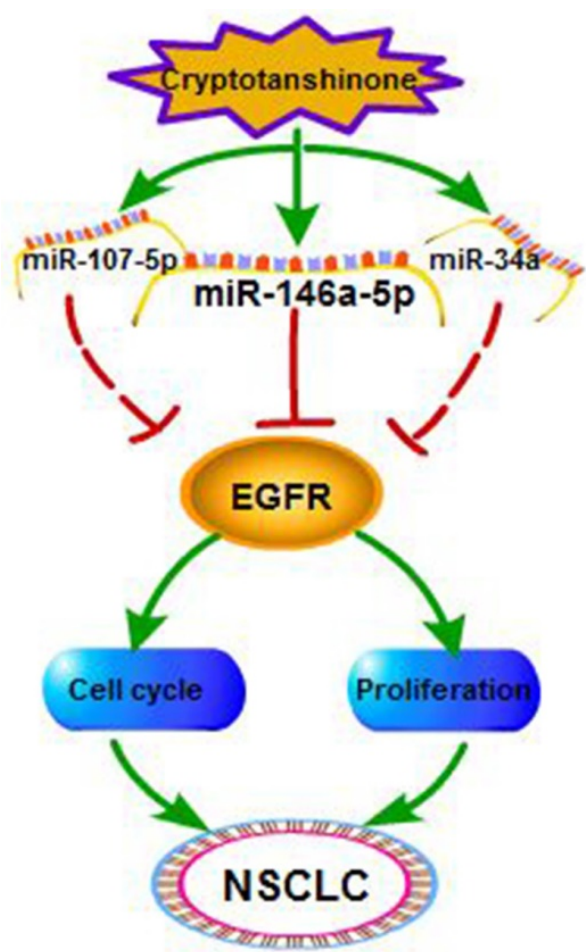

Figure 7. The regulatory network of miR-146a-5p in NSCLC. Our studies suggest Cryptotanshinone suppresses non-small cell lung cancer via miR-146a-5p/EGFR axis.

In previous studies, non-coding RNAs related signaling pathways in the antitumor efficacy of tanshinones were found. It has been proved that miR-137 and miR-32a could be upregulated by tanshinones in NSCLC $[29,30]$. Ren et al. showed T2A induced cell death via miR30b-p53-PTPN11/SHP2 signaling pathway in human hepatocellular carcinoma cells [40]. Moreover, in human esophageal cancer, T2A induced upregulation of miR-122 expression which can suppress cell proliferation via inhibiting pyruvate kinase M2 [41]. Here we showed CT suppressed lung cancer through regulating miR-146a-5p/EGFR axis.

Lung cancer is strongly related to status of EGFR, which is an important target for cancer therapy in precision medicine era. However, the emergence of drug resistance forces us to search for a variety of EGFR targeted therapies. At present, the study of miR-34a as a therapeutic drug for NSCLC has entered the stage of clinical stage I [42]. Studies also found that let-7 and miR-34a could inhibit NSCLC in collaboration with a new generation of EGFR-TKIs,
Erlotinib [43]. Our findings could lead to further understanding of the function of miRNAs in lung cancer. These results indicated miR-146a-5p was a significant tumor inhibitor which might be developed as a drug in the treatment of NSCLC or in combination with EGFR-TKIs.

In conclusion, our study showed that $\mathrm{CT}$ suppresses NSCLC via miR-146a-5p/EGFR axis. Further investigation is required to determine whether CT and miR-146a-5p can as drugs play vital roles in organisms.

\section{Supplementary Material}

Supplementary tables.

http://www.ijbs.com/v15p1072s1.pdf

\section{Acknowledgments}

We are grateful to Professor Xin Lin (Tsinghua University, China) for giving us p2k7-EGFR plasmid. The work was funded and supported by the national natural science foundation (No. 91543123 and 81601887).

\section{Competing Interests}

The authors have declared that no competing interest exists.

\section{References}

1. Bray F, Ferlay J, Soerjomataram I, et al. Global cancer statistics 2018: GLOBOCAN estimates of incidence and mortality worldwide for 36 cancers in 185 countries. CA: a cancer journal for clinicians. 2018.

2. Chen W, Zheng R, Baade PD, et al. Cancer statistics in China, 2015. CA: a cancer journal for clinicians. 2016; 66: 115-32.

3. Siegel RL, Miller KD, Jemal A. Cancer statistics, 2018. CA: a cancer journal for clinicians. 2018; 68: 7-30.

4. Eaton BR, Pugh SL, Bradley JD, et al. Institutional Enrollment and Survival Among NSCLC Patients Receiving Chemoradiation: NRG Oncology Radiation Therapy Oncology Group (RTOG) 0617. Journal of the National Cancer Institute. 2016; 108.

5. Wink KC, Belderbos JS, Dieleman EM, et al. Improved progression free survival for patients with diabetes and locally advanced non-small cell lung cancer (NSCLC) using metformin during concurrent chemoradiotherapy. Radiotherapy and oncology : journal of the European Society for Therapeutic Radiology and Oncology. 2016; 118: 453-9.

6. Liu X, Wang P, Zhang C, et al. Epidermal growth factor receptor (EGFR): A rising star in the era of precision medicine of lung cancer. Oncotarget. 2017; 8: 50209-20.

7. Shostak K, Chariot A. EGFR and NF-kappaB: partners in cancer. Trends in molecular medicine. 2015; 21: 385-93.

8. Linder M, Glitzner E, Srivatsa S, et al. EGFR is required for FOS-dependent bone tumor development via RSK2/CREB signaling. EMBO molecular medicine. 2018.

9. Stichel D, Ebrahimi A, Reuss D, et al. Distribution of EGFR amplification, combined chromosome 7 gain and chromosome 10 loss, and TERT promoter mutation in brain tumors and their potential for the reclassification of IDHwt astrocytoma to glioblastoma. Acta neuropathologica. 2018; 136: 793-803.

10. Jorge SE, Lucena-Araujo AR, Yasuda $\mathrm{H}$, et al. EGFR Exon 20 Insertion Mutations Display Sensitivity to Hsp90 Inhibition in Preclinical Models and Lung Adenocarcinomas. Clinical cancer research : an official journal of the American Association for Cancer Research. 2018.

11. Roncato F, Rruga F, Porcu E, et al. Improvement and extension of anti-EGFR targeting in breast cancer therapy by integration with the Avidin-Nucleic-Acid-Nano-Assemblies. Nature communications. 2018; 9: 4070.

12. Jia Y, Yun CH, Park E, et al. Overcoming EGFR(T790M) and EGFR(C797S) resistance with mutant-selective allosteric inhibitors. Nature. 2016; 534: 129-32.

13. Schrock AB, Frampton GM, Herndon D, et al. Comprehensive Genomic Profiling Identifies Frequent Drug-Sensitive EGFR Exon 19 Deletions in NSCLC not Identified by Prior Molecular Testing. Clinical cancer research : an 
official journal of the American Association for Cancer Research. 2016; 22: 3281-5.

14. Walter AO, Sin RT, Haringsma HJ, et al. Discovery of a mutant-selective covalent inhibitor of EGFR that overcomes T790M-mediated resistance in NSCLC. Cancer discovery. 2013; 3: 1404-15.

15. Aguiar PN, Jr., Haaland B, et al. Cost-effectiveness of Osimertinib in the First-Line Treatment of Patients With EGFR-Mutated Advanced Non-Small Cell Lung Cancer. JAMA oncology. 2018; 4: 1080-4.

16. Beck TN, Kharin LV, Kit OI, et al. Miliary Adenocarcinoma of the Lung Responds to Gefitinib and Afatinib. Journal of thoracic oncology : official publication of the International Association for the Study of Lung Cancer. 2018; 13: e95-e7.

17. Ramalingam SS, Janne PA, Mok T, et al. Dacomitinib versus erlotinib in patients with advanced-stage, previously treated non-small-cell lung cancer (ARCHER 1009): a randomised, double-blind, phase 3 trial. The Lancet Oncology. 2014; 15: 1369-78.

18. Yang JC, Shih JY, Su WC, et al. Afatinib for patients with lung adenocarcinoma and epidermal growth factor receptor mutations (LUX-Lung 2): a phase 2 trial. The Lancet Oncology. 2012; 13: 539-48.

19. Niogret J, Coudert B, Boidot R. Primary Resistance to Afatinib in a Patient with Lung Adenocarcinoma Harboring Uncommon EGFR Mutations: S768I and V769L. Journal of thoracic oncology : official publication of the International Association for the Study of Lung Cancer. 2018; 13: e113.

20. Oxnard GR, Hu Y, Mileham KF, et al. Assessment of Resistance Mechanisms and Clinical Implications in Patients With EGFR T790M-Positive Lung Cancer and Acquired Resistance to Osimertinib. JAMA oncology. 2018.

21. $\mathrm{Xu} \mathrm{H}$, Song J, Luo H, et al. Analysis of the Genome Sequence of the Medicinal Plant Salvia miltiorrhiza. Molecular plant. 2016; 9: 949-52

22. Yu HA, Arcila ME, Rekhtman N, et al. Analysis of tumor specimens at the time of acquired resistance to EGFR-TKI therapy in 155 patients with EGFR-mutant lung cancers. Clinical cancer research : an official journal of the American Association for Cancer Research. 2013; 19: 2240-7.

23. Lu X, Yu L, Zhang $\mathrm{Z}$, et al. Targeting EGFR(L858R/T790M) and EGFR(L858R/T790M/C797S) resistance mutations in NSCLC: Current developments in medicinal chemistry. Medicinal research reviews. 2018; 38: 1550-81.

24. Jia Y, Huang F, Zhang S, et al. Is danshen (Salvia miltiorrhiza) dripping pill more effective than isosorbide dinitrate in treating angina pectoris? A systematic review of randomized controlled trials. International journal of cardiology. 2012; 157: 330-40.

25. Chen W, Luo Y, Liu L, et al. Cryptotanshinone inhibits cancer cell proliferation by suppressing Mammalian target of rapamycin-mediated cyclin D1 expression and $\mathrm{Rb}$ phosphorylation. Cancer prevention research. 2010; 3: 1015-25.

26. Hao $\mathrm{W}$, Zhang $\mathrm{X}$, Zhao $\mathrm{W}$, et al. Cryptotanshinone Induces Pro-death Autophagy through JNK Signaling Mediated by Reactive Oxygen Species Generation in Lung Cancer Cells. Anti-cancer agents in medicinal chemistry. 2016; 16: 593-600.

27. Zhang J, Wen G, Sun L, et al. Cryptotanshinone inhibits cellular proliferation of human lung cancer cells through downregulation ofIGF-1R/PI3K/Akt signaling pathway. Oncology reports. 2018; 40: 2926-34.

28. Xia C, Bai X, Hou X, et al. Cryptotanshinone Reverses Cisplatin Resistance of Human Lung Carcinoma A549 Cells through Down-Regulating Nrf2 Pathway. Cellular physiology and biochemistry : international journal of experimental cellular physiology, biochemistry, and pharmacology. 2015; 37: 816-24.

29. Ma ZL, Zhang BJ, Wang DT, et al. Tanshinones suppress AURKA through up-regulation of miR-32 expression in non-small cell lung cancer. Oncotarget. 2015; 6: 20111-20.

30. Zhang B, Ma Z, Li X, et al. Tanshinones suppress non-small cell lung cancer through up-regulating miR-137. Acta biochimica et biophysica Sinica. 2016; 48 : 768-70.

31. Bartel DP. MicroRNAs: target recognition and regulatory functions. Cell. 2009; 136: 215-33.

32. Li YL, Liu XM, Zhang CY, et al. MicroRNA-34a/EGFR axis plays pivotal roles in lung tumorigenesis. Oncogenesis. 2017; 6: e372.

33. Mori M, Triboulet R, Mohseni M, et al. Hippo signaling regulates microprocessor and links cell-density-dependent miRNA biogenesis to cancer. Cell. 2014; 156: 893-906.

34. He Y, Xu H, Li C, et al. Nicastrin/miR-30a-3p/RAB31 Axis Regulates Keratinocyte Differentiation by Impairing EGFR Signaling in Familial Acne Inversa. The Journal of investigative dermatology. 2018.

35. Migliore C, Morando E, Ghiso E, et al. miR-205 mediates adaptive resistance to MET inhibition via ERRFI1 targeting and raised EGFR signaling. EMBO molecular medicine. 2018; 10.

36. Liu R, Yi B, Wei S, et al. FOXP3-miR-146-NF-kappaB Axis and Therapy for Precancerous Lesions in Prostate. Cancer research. 2015; 75: 1714-24.

37. Li YL, Wang J, Zhang CY, et al. MiR-146a-5p inhibits cell proliferation and cell cycle progression in NSCLC cell lines by targeting CCND1 and CCND2. Oncotarget. 2016; 7: 59287-98.

38. Wang P, Liu X, Shao Y, et al. MicroRNA-107-5p suppresses non-small cell lung cancer by directly targeting oncogene epidermal growth factor receptor. Oncotarget. 2017; 8: 57012-23.
39. Fang J, Little PI, Xu S. Atheroprotective Effects and Molecular Targets of Tanshinones Derived From Herbal Medicine Danshen. Medicinal research reviews. 2018; 38: 201-28.

40. Ren X, Wang C, Xie B, et al. Tanshinone IIA induced cell death via miR30b-p53-PTPN11/SHP2 signaling pathway in human hepatocellular carcinoma cells. European journal of pharmacology. 2017; 796: 233-41.

41. Zhang HS, Zhang FJ, $\mathrm{Li} \mathrm{H}$, et al. Tanshinone A inhibits human esophageal cancer cell growth through miR-122-mediated PKM2 down-regulation. Archives of biochemistry and biophysics. 2016; 598: 50-6.

42. Misso G, Di Martino MT, De Rosa G, et al. Mir-34: a new weapon against cancer? Molecular therapy Nucleic acids. 2014; 3: e194.

43. Stahlhut C, Slack FJ. Combinatorial Action of MicroRNAs let-7 and miR-34 Effectively Synergizes with Erlotinib to Suppress Non-small Cell Lung Cancer Cell Proliferation. Cell cycle. 2015; 14: 2171-80. 